\title{
Actuator cylinder theory for multiple vertical axis wind turbines
}

\author{
Andrew Ning \\ Brigham Young University, Provo, UT, USA \\ Correspondence to: Andrew Ning (aning@byu.edu)
}

\author{
Received: 13 May 2016 - Published in Wind Energ. Sci. Discuss.: 31 May 2016 \\ Revised: 15 September 2016 - Accepted: 7 November 2016 - Published: 16 December 2016
}

\begin{abstract}
Actuator cylinder theory is an effective approach for analyzing the aerodynamic performance of vertical axis wind turbines at a conceptual design level. Existing actuator cylinder theory can analyze single turbines, but analysis of multiple turbines is often desirable because turbines may operate in near proximity within a wind farm. For vertical axis wind turbines, which tend to operate in closer proximity than do horizontal axis turbines, aerodynamic interactions may not be strictly confined to wake interactions. We modified actuator cylinder theory to permit the simultaneous solution of aerodynamic loading for any number of turbines. We also extended the theory to handle thrust coefficients outside of the momentum region and explicitly defined the additional terms needed for curved or swept blades.

While the focus of this paper is a derivation of an extended methodology, an application of this theory was explored involving two turbines operating in close proximity. Comparisons were made against two-dimensional unsteady Reynolds-averaged Navier-Stokes (URANS) simulations, across a full $360^{\circ}$ of inflow, with excellent agreement. The counter-rotating turbines produced a 5-10\% increase in power across a wide range of inflow conditions. A second comparison was made to a three-dimensional RANS simulation with a different turbine under different conditions. While only one data point was available, the agreement was reasonable, with the computational fluid dynamics (CFD) predicting a $12 \%$ power loss, as compared to a $15 \%$ power loss for the actuator cylinder method. This extended theory appears promising for conceptual design studies of closely spaced vertical axis wind turbines (VAWTs), but further development and validation is needed.
\end{abstract}

\section{Introduction}

Blade element momentum theory combines momentum theory across an actuator disk with blade element theory to predict the aerodynamic loading of horizontal axis wind turbines (HAWTs). This theory has been very successful and is heavily used in many analysis and design applications (Hansen, 2008; Manwell et al., 2009; Burton et al., 2011; Ning, 2014). Its primary advantage is computational speed while still providing reasonably accurate performance predictions.

Streamtube theory attempts to apply the same concept to vertical axis wind turbine (VAWT) aerodynamic performance estimation (Templin, 1974). Each cross section of the VAWT (constant height) is approximated as an actuator disk through the mid-plane, which results in a crossplane actuator line in the 2-D plane. However, this model is a rather poor representation of a VAWT as it requires constant flow parameters across the entire disk. An extension of this theory is multiple streamtube theory, where, instead of using one large streamtube passing through the VAWT, the VAWT cross section is discretized into multiple streamtubes each with an independent induction factor (Wilson and Lissaman, 1974; Strickland, 1975). An additional extension, double multiple streamtube theory (Paraschivoiu, 1981, 1988; Paraschivoiu and Delclaux, 1983), utilizes two actuator disks to represent the upstream and downstream sides of the cylinder (Fig. 1). In this model, momentum losses can occur on both the upwind and downwind faces. Double multiple streamtube theory has been widely used for aerodynamic analysis of VAWTs. 


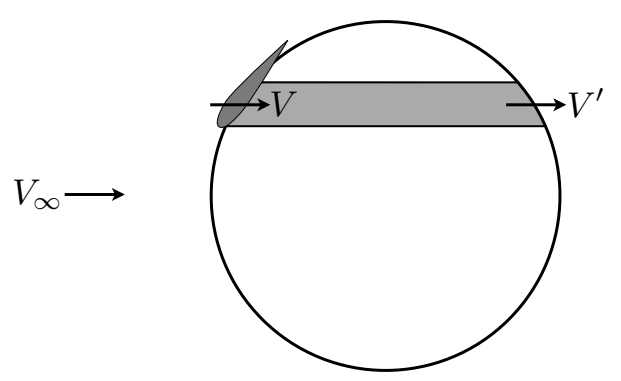

Figure 1. Double multiple streamtube concept with multiple streamtubes along the VAWT (one shown) and separate "actuator disks" on both the upstream and downstream surfaces.

While double multiple streamtube theory is a useful improvement over single streamtube models, it is clearly a forced application of the actuator disk concept to a VAWT. A more physically consistent theory for VAWTs, called actuator cylinder theory, was developed by Madsen (Madsen, 1982; Madsen et al., 2013). Actuator cylinder theory has been shown to be more accurate than double multiple streamtube theory (Ferreira et al., 2014), while still retaining comparable computational speed.

One limitation of actuator cylinder theory is that it is derived only for a single isolated turbine. We are interested in performance of VAWT farms and thus need to predict performance of multiple VAWTs in proximity to each other. This paper extends the methodology for use with any number of VAWTs, extends applicability to turbines not operating in the momentum region, and adds computation details for blades that are curved or swept. The primary purpose of this paper is to derive the new methodology, but some example trade studies of VAWT pairs are also discussed.

\section{Theory development}

The actuator cylinder theory begins with the assumption that a vertical slice of a VAWT can be modeled as a twodimensional problem. Figure 2 shows a 2-D representation of the VAWT, with only one of the blades shown for simplicity, and defines the coordinate system used in this derivation. The VAWT produces a varying normalized radial force per unit length $q(\theta)$ as a function of azimuthal position along the VAWT. We define the positive direction for this force $q$ as positive radial outward (and thus positive radially inward for the loads the fluid produces on the VAWT). Using the two-dimensional, steady, incompressible, Euler equations, and (for the moment) neglecting nonlinear terms, the induced velocities at any location in the plane can be shown to be given by the following integrals (Madsen et al., 2013; Madsen, 1982):

$u(x, y)=\frac{1}{2 \pi} \int_{0}^{2 \pi} q(\theta) \frac{[x+\sin \theta] \sin \theta-[y-\cos \theta] \cos \theta}{[x+\sin \theta]^{2}+[y-\cos \theta]^{2}} \mathrm{~d} \theta$

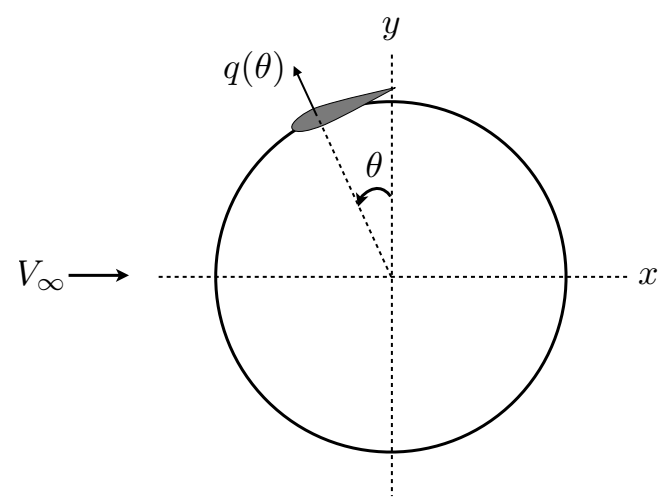

Figure 2. A canonical 2-D slice of a VAWT (only one blade shown) and the coordinate system used.

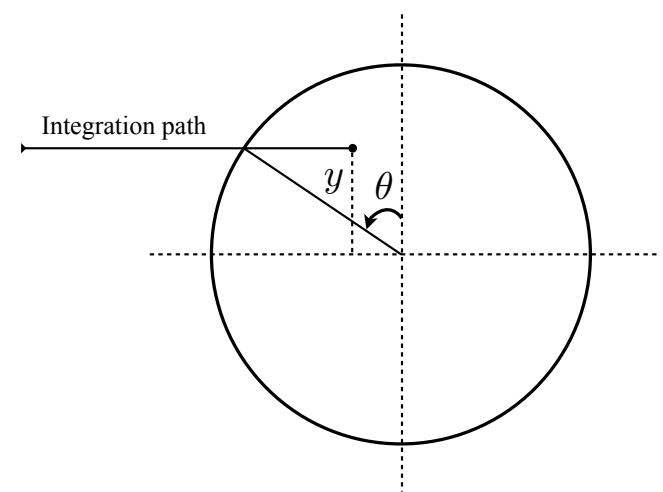

Figure 3. Integration path for a point inside the cylinder.

$$
\begin{aligned}
& \left.-q\left(\cos ^{-1} y\right) \quad \text { inside and wake }\right\} \\
& +q\left(-\cos ^{-1} y\right) \quad\{\text { wake only }
\end{aligned}
$$

$v(x, y)=\frac{1}{2 \pi} \int_{0}^{2 \pi} q(\theta) \frac{[x+\sin \theta] \cos \theta+[y-\cos \theta] \sin \theta}{[x+\sin \theta]^{2}+[y-\cos \theta]^{2}} \mathrm{~d} \theta$,

where the $x, y$ position is measured from the center of a unit radius turbine, and velocities are normalized by the freestream velocity. For evaluation points inside the cylinder the \{inside and wake\} term applies, and for evaluation points downstream of the cylinder both the \{inside and wake\} and \{wake only\} terms apply. These two terms are based on an integration path through the cylinder, where $\theta=\cos ^{-1} y$ (Fig. 3). For brevity, the derivations of the above equations are omitted, but details are available in the above-cited papers from Madsen.

These two equations for the induced velocities (Eq. 1) are applicable for any $x, y$ location; however, we are primarily interested in the induced velocities only at locations on the current turbine and on other turbines. To facilitate computation we discretize the description of each actuator cylinder 
into $n$ panels centered at the azimuthal locations:

$$
\begin{aligned}
\theta_{i} & =(2 i-1) \frac{\pi}{n} \text { for } i=1 \ldots n, \\
\Delta \theta & =\frac{2 \pi}{n} .
\end{aligned}
$$

Furthermore, as is done in the original version, we assume piecewise constant loading across each panel. These locations are the points of interest where will compute the radial forces and subsequently the induced velocities.

In general, we need to compute the induced velocity at every location on a given VAWT using contributions from all VAWTs (including itself). In the following derivation we adopt the notation that index $I$ is the turbine we are evaluating the velocities at, and index $i$ represents the azimuthal location on turbine $I$ where we are evaluating. Index $J$ will refer to the turbine producing the induced velocity, and index $j$ will indicate the azimuthal location on turbine $J$ where the load is producing the induced velocity (Fig. 4).

Using the azimuthal discretization, the induced velocities at a point $(x, y)$ are expressed as a sum of integrals over individual panels. Recall that Eq. (1) is normalized based on the current VAWT radius and the freestream velocity. Because we are now considering multiple VAWTs with potentially different radii, we need to be more explicit in defining the normalized quantities. The generalized definitions of the $x, y$ evaluation positions are

$x_{i}^{*}=\left(x_{i}-x_{J_{\mathrm{c}}}\right) / r_{J}$,

$y_{i}^{*}=\left(y_{i}-y_{J_{\mathrm{c}}}\right) / r_{J}$,

where $x_{J_{\mathrm{c}}}$ is the $x$ location of the center of turbine $J$. If $I=J$ (i.e., we are evaluating the turbine's influence on itself), then this definition is identical to the single-turbine case where the $x$ and $y$ locations are then distances from the VAWT center normalized by its radius.

The velocity used in normalizing the induced velocities and the radial loading must be the same, and for that purpose we continue to use the freestream velocity. We introduce the asterisk superscript on the induced velocities for clarity (e.g., $u^{*}=u / V_{\infty}$ ). The expressions for induced velocity at the cylinder surface depend on whether we evaluate just upstream of the actuator disk or just downstream. The end result is the same, as long as we are consistent. In the following derivation we evaluate on the upstream surfaces for both halves of the actuator disk.

$$
\begin{aligned}
u_{i}^{*}= & \frac{1}{2 \pi} \sum_{j} q_{j} \int_{\theta_{j}-\Delta \theta / 2}^{\theta_{j}+\Delta \theta / 2} \\
& \frac{\left(x_{i}^{*}+\sin \phi\right) \sin \phi-\left(y_{i}^{*}-\cos \phi\right) \cos \phi}{\left(x_{i}^{*}+\sin \phi\right)^{2}+\left(y_{i}^{*}-\cos \phi\right)^{2}} \mathrm{~d} \phi \\
& -q_{n+1-i} \quad\{I=J, i>n / 2\} \\
& -q_{J_{k}}+q_{J_{n+1-k}} \quad\left\{I \neq J,-1 \leq y_{i}^{*} \leq 1, x_{i}^{*} \geq 1\right\}
\end{aligned}
$$

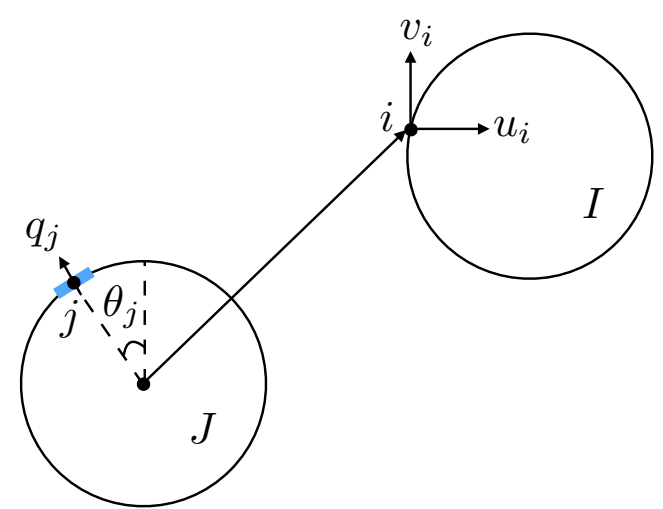

Figure 4. Influence of load at location $j$ of turbine $J$ onto location $i$ of turbine $I$.

$$
\begin{aligned}
& \left(\text { where index } k \text { satisfies } \theta_{J_{k}}=\cos ^{-1} y_{i}^{*}\right) \\
v_{i}^{*}= & \frac{1}{2 \pi} \sum_{j} q_{j} \int_{\theta_{j}-\Delta \theta / 2}^{\theta_{j}+\Delta \theta / 2} \\
& \frac{\left(x_{i}^{*}+\sin \phi\right) \cos \phi+\left(y_{i}^{*}-\cos \phi\right) \sin \phi}{\left(x_{i}^{*}+\sin \phi\right)^{2}+\left(y_{i}^{*}-\cos \phi\right)^{2}} \mathrm{~d} \phi
\end{aligned}
$$

In these integrals we have replaced $\theta$ in the integration with the dummy variable $\phi$ in order to avoid confusion with the $\theta$ terms appearing in the integration limits. The term $-q_{n+1-i}$ arises when evaluating the influence of a turbine on itself. Because we chose to evaluate on the upstream surfaces, the upstream half of the VAWT is considered outside of the VAWT, but the aft half is in the inside of the cylinder. This implies that for the aft half (i.e., $i>n / 2)$ the $-q\left(\cos ^{-1} y\right)$ term must be added. This corresponds to the loading on the front half of the turbine with the same $y$ value. Based on our discretization, its location can be indexed directly as $-q_{n+1-i}$.

The following two terms for $u$ arise when turbine $I$ is in the wake of turbine $J$. Actuator cylinder theory only includes the wake term when an evaluation point is directly downwind from a source point (e.g., the blue region in Fig. 5). The condition corresponds to $x_{i}^{*} \geq 1$ and $-1 \leq y_{i}^{*} \leq 1$ and $x_{i}^{* 2}+y_{i}^{* 2} \geq 1$. For this wake area, both of the terms in Eq. 1 are applicable. The index $k$ corresponds to the location where $\theta_{J_{k}}=\cos ^{-1} y_{i}^{*}$. Note that $\cos ^{-1} y_{i}^{*}$ will likely not line up exactly with an existing grid point $\theta_{k}$ on turbine $J$, but we have assumed piecewise constant loading across a given panel, so $k$ will correspond to the panel that is intersected.

This model is based on integration paths like those shown in Fig. 3 and thus ignores the effect of wake expansion and viscous decay. An alternative is to ignore the wake terms and instead apply a momentum deficit factor from some other VAWT wake model. For example, we have developed a reduced-order wake model based on computational fluid dynamics (CFD) simulations that predicts the velocity deficit behind a VAWT (Tingey and Ning, 2016). Rather than use 


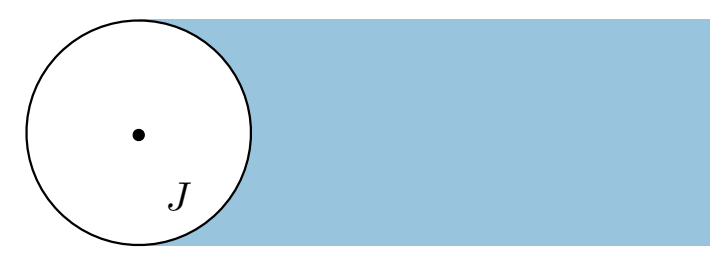

Figure 5. Wake region from actuator cylinder theory highlighted in blue (and extending downstream).

the above wake terms, one could use the separate wake model to evaluate the velocity deficit at each control point and subtract the deficit from $\boldsymbol{u}^{*}$. Because the focus on this paper is on actuator cylinder theory, we will use the simple wake model that naturally arises within the theory itself, but this methodology provides a convenient hook to insert any wake model.

For convenience in the computation, Eq. (4) can be expressed as a matrix vector multiplication where the loading $\boldsymbol{q}$ is separated from the influence coefficients.

$\boldsymbol{u}_{I}^{*}=\mathbf{A}_{x_{I J}} \boldsymbol{q}_{J}$

$\boldsymbol{v}_{I}^{*}=\mathbf{A}_{y_{I J}} \boldsymbol{q}_{J}$

The matrix $\mathbf{A}_{y_{I J}}$ is given by

$$
\begin{aligned}
& \mathbf{A}_{y_{I} J}(i, j)=\frac{1}{2 \pi} \int_{\theta_{j}-\Delta \theta / 2}^{\theta_{j}+\Delta \theta / 2} \\
& \frac{\left(x_{i}^{*}+\sin \phi\right) \cos \phi+\left(y_{i}^{*}-\cos \phi\right) \sin \phi}{\left(x_{i}^{*}+\sin \phi\right)^{2}+\left(y_{i}^{*}-\cos \phi\right)^{2}} \mathrm{~d} \phi .
\end{aligned}
$$

For the $\mathbf{A}_{x_{I J}}$ matrix we divide the contributions between the direct influence and the wake influence: $\mathbf{A}_{x_{I J}}=\mathbf{D}_{x_{I J}}+$ $\mathbf{W}_{x_{I J}}$, where

$$
\begin{aligned}
& \mathbf{D}_{x_{I J}}(i, j)=\frac{1}{2 \pi} \int_{\theta_{j}-\Delta \theta / 2}^{\theta_{j}+\Delta \theta / 2} \\
& \frac{\left(x_{i}^{*}+\sin \phi\right) \sin \phi-\left(y_{i}^{*}-\cos \phi\right) \cos \phi}{\left(x_{i}^{*}+\sin \phi\right)^{2}+\left(y_{i}^{*}-\cos \phi\right)^{2}} \mathrm{~d} \phi,
\end{aligned}
$$

$\mathbf{W}_{x_{I J}}(i, j)=\left\{\begin{aligned}-1 & \text { if }-1 \leq y_{i}^{*} \leq 1 \text { and } x_{i}^{*} \geq 0 \\ & \text { and } x_{i}^{* 2}+y_{i}^{* 2} \geq 1 \text { and } j=k \\ 1 & \text { if }-1 \leq y_{i}^{*} \leq 1 \text { and } x_{i}^{*} \geq 0 \\ & \text { and } x_{i}^{* 2}+y_{i}^{* 2} \geq 1 \\ & \text { and } j=n-k+1 \\ 0 & \text { otherwise, }\end{aligned}\right.$

where index $k$ corresponds to the panel where $\theta_{J_{k}}=$ $\cos ^{-1} y_{i}^{*}$.

If we are evaluating the influence of a turbine on itself (e.g., $I=J$ ) then the computations in the $\mathbf{A}_{x}$ matrix can be simplified. We can expand using the definitions for $x$ and $y$ along the cylinder $\left(x_{i}^{*}=-\sin \theta_{i}\right.$ and $y_{i}^{*}=\cos \theta_{i}$ for $i=1 \ldots n)$. As long as $i \neq j$, then the integral in Eq. (7) evaluates to $\Delta \theta / 2$. When $i=j$ the value of the integral depends on which side of the cylinder we evaluate on. It converges to $\pi(-1+1 / n)$ just outside of the cylinder and $\pi(1+1 / n)$ just inside. Because we chose to evaluate on the upstream surface on both halves of the cylinder, the integral evaluates to $\pi(-1+1 / n)$ on the upstream half of the cylinder and $\pi(1+1 / n)$ on the downstream half of the cylinder.

$\mathbf{D}_{x_{I I}}(i, j)= \begin{cases}\Delta \theta /(4 \pi) & \text { if } i \neq j \\ (-1+1 / n) / 2 & \text { if } i=j \text { and } i \leq n / 2 \\ (1+1 / n) / 2 & \text { if } i=j \text { and } i>n / 2\end{cases}$

$\mathbf{W}_{x_{I I}}(i, j)=\left\{\begin{array}{ll}-1 & \text { if } i>n / 2 \\ 0 & \text { otherwise }\end{array}\right.$ and $j=n+1-i$

If a user elects to use a more sophisticated wake model the $\mathbf{W}_{x}$ term can simply be ignored and a separate momentum deficit factor can be applied.

\subsection{Faster computation}

The bulk of the computational effort is contained in computing the influence coefficient matrices $\mathbf{A}_{x_{I J}}$ and $\mathbf{A}_{y_{I J}}$. These computations consist of a double loop iterating across all evaluation positions $i$ on turbine $I$ for each source position $j$ on turbine $J$ (which is itself contained in a double loop across all turbines $I$ and $J$ ). Fortunately, some of this computation can be simplified. The expressions in Eqs. (6), (9), and (10) apply for the cases where $I=J$, or in other words for computing the influence of the turbine on itself. A significant benefit to this equation form is that the matrices depend only on the discretization of the cylinder and not on the details of the blade shape or loading. For a preselected number of azimuthal segments (e.g., $n=36$ ), these matrices can be precomputed and stored. This is true no matter what size radius the VAWT is.

If $I \neq J$, some reduction in computational requirements is also possible. For each VAWT pair $(I \neq J)$, if the two VAWTs are of equal radius, then pairs of influence coefficients between them are exactly the same. As seen in Fig. 6, the distance vector from the center of one turbine to the evaluation point on a separate turbine is exactly equal and opposite to a vector originating from the center of the other turbine and terminating at an azimuthal location diametrically opposite to the first evaluation point's azimuthal location. As long as these two VAWTs are of equal radius, these two vectors will always be equal and opposite. This corresponds to $\boldsymbol{x}^{*}$ and $\boldsymbol{y}^{*}$ switching signs in Eqs. (6) and (7). However, the evaluation locations are always $180^{\circ}$ apart in location. This corresponds to switching the sign on all sin and cos terms. The two sign changes cancel out and thus the two evaluation coefficients will be exactly the same. In other words, for all pairs of VAWTs that are of equal radii, only one set of 


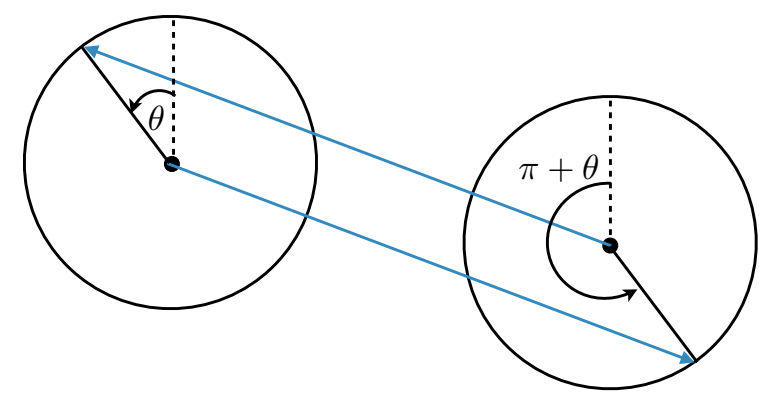

Figure 6. The influence coefficient calculations between a pair of VAWTs will always have paired locations that have exactly equal and opposite distance vectors if the two VAWTs are of equal radius. These two evaluation locations result in the exact same influence coefficients, reducing the amount of calculations that must be performed.

influence coefficients need be computed. The influence coefficients for the other VAWT can be mapped over directly. In equation form this is given by

$$
\begin{aligned}
& \mathbf{D}_{x_{J I}}((i+n / 2) \bmod n,(j+n / 2) \bmod n) \\
& \quad=\mathbf{D}_{x_{I J}}(i, j), \quad \forall i=1 \ldots n, j=1 \ldots n\left(\text { if } r_{I}=r_{J}\right)
\end{aligned}
$$

and similarly for $A_{y}$. Note that there is no symmetry in the wake terms (Eq. 8). If a second turbine is in the wake of the first, the first turbine will clearly not be in the wake of the second turbine.

Finally, we can reduce the number of computations required for VAWTs that have large separation distances. If a VAWT pair has a large separation distance (e.g., $\left.\sqrt{\left(x_{I_{\mathrm{c}}}-x_{J_{\mathrm{c}}}\right)^{2}+\left(y_{I_{\mathrm{c}}}-y_{J_{\mathrm{c}}}\right)^{2}}>10 r_{I}\right)$, then when iterating across index $i$ the value for positions $x_{i}$ and $y_{i}$ will change very little. The computation can be simplified by neglecting these very minor changes and instead using the distance between VAWT centers (independent of $i$ ):

$x_{i}^{*} \rightarrow\left(x_{I_{\mathrm{c}}}-x_{J_{\mathrm{c}}}\right) / r_{J}$,

$y_{i}^{*} \rightarrow\left(y_{I_{\mathrm{c}}}-y_{J_{\mathrm{c}}}\right) / r_{J}$.

With this simplification the matrices in Eqs. (6) and (7) can be computed by iterating only in $j$ and filling an entire column per iteration. Additionally, for these large separations the wake terms should be negligible and can be skipped in the computation.

\subsection{Body forces}

With the induced velocities $\boldsymbol{u}^{*}$ and $\boldsymbol{v}^{*}$, we can compute the body forces produced by the VAWT. The volume forces produced by the VAWT are modeled as acting along an infinitesimally small radial distance and in a direction normal to the surface of the cylinder (the tangential component is much smaller than the normal force and can be reasonably neglected in the volume forces of the Euler equations). The

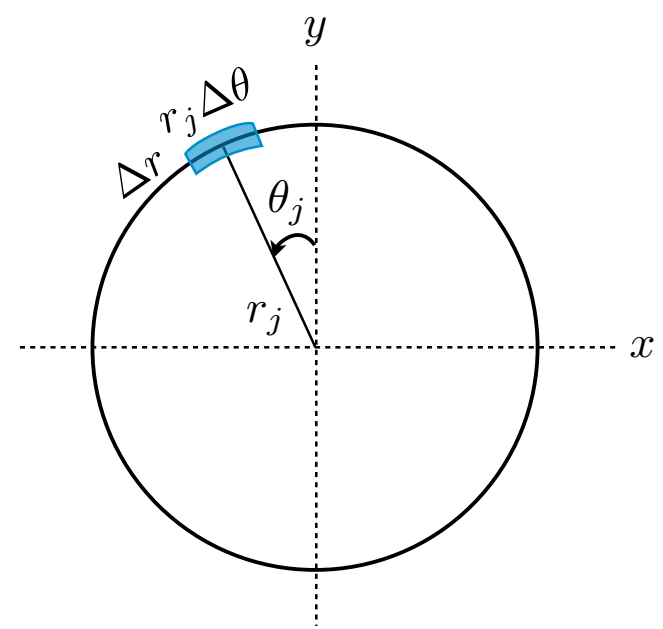

Figure 7. In-plane area for volume force at a given azimuthal station.

radial volume force is

$f_{\mathrm{r}}(\theta)=\frac{F_{\mathrm{r}}^{\prime}}{r_{j} \Delta \theta \Delta r} \frac{L}{\rho V_{\infty}^{2}}$,

where $F_{\mathrm{r}}^{\prime}$ is an azimuthally averaged radial force per unit length in a direction pointing into the center of the cylinder, $r_{j}$ is the radius of the local VAWT cross section, and $r_{j} \Delta \theta \Delta r$ is the in-plane area across which the force acts (Fig. 7). The last term comes from the normalization of the Euler equations, where $L$ is some relevant length scale.

Because the force acts across an infinitesimal small radial distance, the radial force acts as a pressure jump

$$
\begin{aligned}
q(\theta) & =\lim _{\epsilon \rightarrow 0} \frac{1}{L} \int_{r_{j}-\epsilon}^{r_{j}+\epsilon} f_{\mathrm{r}}(\theta) \mathrm{d} r \\
& =\lim _{\epsilon \rightarrow 0} \frac{1}{L} \int_{r_{j}-\epsilon}^{r_{j}+\epsilon} \frac{F_{\mathrm{r}}^{\prime}}{r_{j} \Delta \theta \mathrm{d} r} \frac{L}{\rho V_{\infty}^{2}} \mathrm{~d} r, \\
& =\frac{F_{\mathrm{r}}^{\prime}}{r_{j} \Delta \theta} \frac{1}{\rho V_{\infty}^{2}} .
\end{aligned}
$$

Here, the $1 / L$ is necessary to be consistent with the normalization. It does not matter which reference length is used in normalizing $q(\theta)$ because the length scales cancel.

Figure 8 shows the relative components of velocity in the frame of the airfoil. It consists of contributions from the freestream velocity, the velocity due to rotation, and the induced velocities from itself and other turbines.

$$
\boldsymbol{V}_{j}=V_{\infty}\left(1+u_{j}\right) \hat{x}+V_{\infty} v_{j} \hat{y}-\Omega_{j} r_{j} \hat{t}
$$




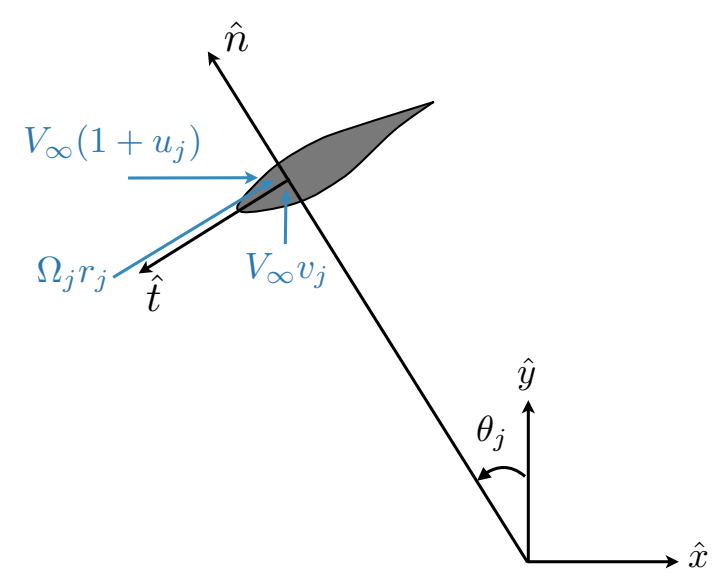

Figure 8. Relative components of velocity in the frame of the airfoil.

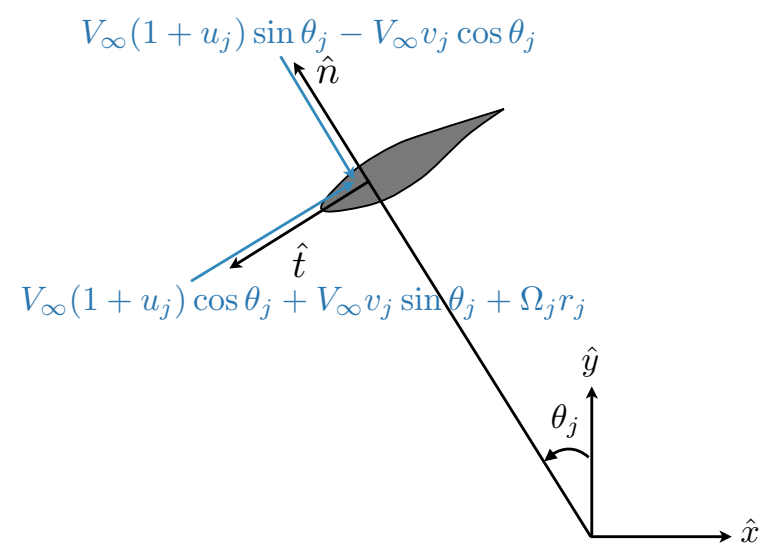

Figure 9. Components of velocity resolved into $\hat{n}-\hat{t}$ plane.

Using the following coordinate transformations,

$\hat{\boldsymbol{x}}=-\cos \theta_{j} \hat{\boldsymbol{t}}-\sin \theta_{j} \hat{\boldsymbol{n}}$

$\hat{\boldsymbol{y}}=-\sin \theta_{j} \hat{\boldsymbol{t}}+\cos \theta_{j} \hat{\boldsymbol{n}}$,

the velocity can be expressed in the $\hat{n}-\hat{t}$ plane as

$$
\begin{aligned}
\boldsymbol{V}_{j} & =\left[-V_{\infty}\left(1+u_{j}\right) \sin \theta_{j}+V_{\infty} v_{j} \cos \theta_{j}\right] \hat{\boldsymbol{n}} \\
& +\left[-V_{\infty}\left(1+u_{j}\right) \cos \theta_{j}-V_{\infty} v_{j} \sin \theta_{j}-\Omega_{j} r_{j}\right] \hat{\boldsymbol{t}} .
\end{aligned}
$$

These velocity components are depicted in Fig. 9.

If we define the magnitudes

$$
\begin{aligned}
V_{\mathrm{n}_{j}} & \equiv V_{\infty}\left(1+u_{j}\right) \sin \theta-V_{\infty} v_{j} \cos \theta, \\
V_{\mathrm{t}_{j}} & \equiv V_{\infty}\left(1+u_{j}\right) \cos \theta+V_{\infty} v_{j} \sin \theta+\Omega_{j} r_{j},
\end{aligned}
$$

then

$\boldsymbol{V}_{j}=-V_{\mathrm{n}_{j}} \hat{n}-V_{\mathrm{t}_{j}} \hat{t}$

and the magnitude of the local relative velocity and local inflow angle (Fig. 10) are

$W_{j}=\sqrt{V_{\mathrm{n}_{j}}^{2}+V_{\mathrm{t}_{j}}^{2}}$,

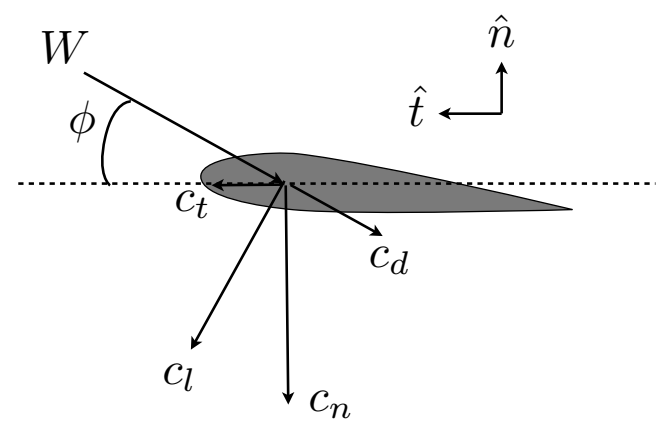

Figure 10. Definition of normal and tangential force coefficients.

$$
\phi_{j}=\tan ^{-1}\left(\frac{V_{\mathrm{n}_{j}}}{V_{\mathrm{t}_{j}}}\right) .
$$

The angle of attack, Reynolds number, and lift and drag coefficients can then be estimated as

$$
\begin{aligned}
\alpha_{j} & =\phi_{j}-\beta \\
R e_{j} & =\frac{\rho W_{j} c}{\mu} \\
c_{1_{j}} & =f\left(\alpha_{j}, R e_{j}\right) \\
c_{\mathrm{d}_{j}} & =f\left(\alpha_{j}, R e_{j}\right) .
\end{aligned}
$$

This can be rotated into normal and tangential force coefficients (note that $c_{n}$ is defined as positive in the opposite direction of $\hat{n}$ in Fig. 10).

$\begin{aligned} c_{\mathrm{n}_{j}} & =c_{1_{j}} \cos \phi_{j}+c_{\mathrm{d}_{j}} \sin \phi_{j} \\ c_{\mathrm{t}_{j}} & =c_{1_{j}} \sin \phi_{j}-c_{\mathrm{d}_{j}} \cos \phi_{j}\end{aligned}$

We can resolve these normal and tangential loads into a radial, tangential, and vertical coordinate system. In doing so, we will account for blade curvature, as is often used with VAWTs, an example of which is shown in Fig. 11. The total force vector is resolved as

$F=\frac{1}{2} \rho W^{2}\left(-c_{\mathrm{n}} \hat{\boldsymbol{n}}+c_{\mathrm{t}} \hat{\boldsymbol{t}}\right) \Delta a$,

where the negative sign results from the coordinate system definition seen in Fig. 10. From Fig. 11 we see that the area of the blade element is

$\Delta a=c \Delta s=c \frac{\Delta z}{\cos \delta}$,

and the unit vector $\hat{\boldsymbol{n}}$ can be expressed as

$\hat{\boldsymbol{n}}=\cos \delta \hat{\boldsymbol{r}}+\sin \delta \hat{\boldsymbol{z}}$.

Thus, the force vector per unit depth (unit length in the $z$ direction) is

$\boldsymbol{F}^{\prime}=\frac{\rho W^{2} c}{2 \cos \delta}\left(-c_{\mathrm{n}} \cos \delta \hat{\boldsymbol{r}}-c_{\mathrm{n}} \sin \delta \hat{\boldsymbol{z}}+c_{\mathrm{t}} \hat{\boldsymbol{t}}\right)$. 


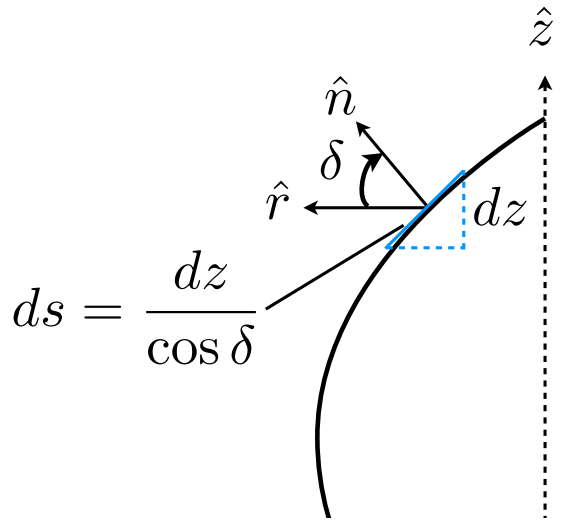

Figure 11. Cross-sectional length of blade segment for small changes in height. Blade curvature increases the area of the blade element for unit height, but sweep has no effect on the blade element area as it is a shearing operation.

We can simplify these expressions for the three instantaneous force components:

$R^{\prime}=-c_{\mathrm{n}} \frac{1}{2} \rho W^{2} c$

$T^{\prime}=c_{\mathrm{t}} \frac{1}{2} \rho W^{2} \frac{c}{\cos \delta}$

$Z^{\prime}=-c_{\mathrm{n}} \frac{1}{2} \rho W^{2} c \tan \delta$.

Note that the radial force is unaffected by blade curvature because although the in-plane normal force varies with the cosine of the local curvature angle $\delta$ (Fig. 11), the area over which the force acts varies inversely with the cosine of the angle. Blade sweep is also permitted; however, it is assumed that the sweep is accomplished through shearing rather than rotation. In other words, it assumed that the airfoils are still defined relative to the streamwise direction as opposed to normal to the local blade sweep. Thus, sweeping does not increase the area of the blade element.

For equating with the actuator cylinder theory, only the radial force is of interest (but all components will be of use for computing overall power and loads). Because the blades are rotating we need to compute an azimuthally averaged value of the radial loading (recalling that the sign convention for a positive radial loading is inward for loads the fluid produces on the VAWT):

$F_{\mathrm{r}_{j}}^{\prime}=c_{\mathrm{n}_{j}} \frac{1}{2} \rho W_{j}^{2} c \frac{B \Delta \theta}{2 \pi}$.

Substituting this radial loading into Eq. (14), we find that the radial volume force can be expressed as

$q_{j}=c_{\mathrm{n}_{j}} \frac{1}{2} \rho W_{j}^{2} c \frac{B \Delta \theta}{2 \pi} \frac{1}{r_{j} \Delta \theta} \frac{1}{\rho V_{\infty}^{2}}$.
After simplification, the radial force is

$q_{j}=\frac{B c}{4 \pi r_{j}} c_{\mathrm{n}_{j}}\left(\frac{W_{j}}{V_{\infty}}\right)^{2}$.

Defining solidity as is typically done for a VAWT $(\sigma=$ $B c / r$ ), the normalized radial force per unit length becomes

$q_{j}=\frac{1}{4 \pi} \sigma_{j} c_{\mathrm{n}_{j}}\left(\frac{W_{j}}{V_{\infty}}\right)^{2}$.

\subsection{Correction factor}

Madsen et al. (2013) note that the induced velocities from the linear solution fit well at low loading, and at high loading they produce good trends but with significant error in overall magnitude. For a uniform loading across a 2-D actuator disk, this linear solution can be shown to produce the following relationship between the thrust coefficient and the induction factor $\left(a=-u / V_{\infty}\right)$ :

$C_{\mathrm{T}_{\text {linear }}}=4 a_{\text {linear }}$.

We can equate this thrust coefficient prediction to that of blade element momentum theory in order to produce a correction factor for $a_{\text {linear. }}$. We extend the approach used by Madsen to consider more than just the momentum region. The relationship between the thrust coefficient and the induction factors varies more generally depending on the induction factor (Wilson and Lissaman, 1974; Buhl Jr., 2005):

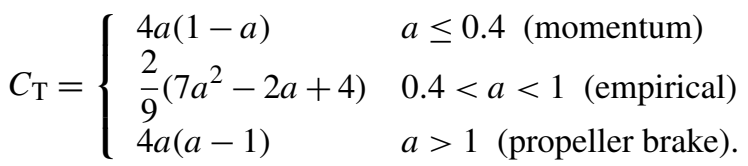

In order to get the same induction factor from the linear solution, as would be predicted by blade element momentum theory, we need to multiply our predicted induced velocities (and thus the thrust coefficient) by the correction factor $k_{a}=C_{\mathrm{T}_{\text {linear }}} / C_{\mathrm{T}}$.

The correction factors become

$k_{a}= \begin{cases}1 /(1-a) & (\text { momentum) } \\ (18 a) /\left(7 a^{2}-2 a+4\right) & \text { (empirical) } \\ 1 /(a-1) & \text { (propeller brake). }\end{cases}$

In order to determine the value of $a$ to use in the above equation we first find the thrust coefficient. The instantaneous thrust coefficient can be found from Eq. (27) using the coordinate system definition that

$$
\begin{aligned}
X^{\prime} & =-R^{\prime} \sin \theta-T^{\prime} \cos \theta \\
& =\frac{1}{2} \rho W^{2} c\left(c_{\mathrm{n}} \sin \theta-c_{\mathrm{t}} \frac{\cos \theta}{\cos \delta}\right) .
\end{aligned}
$$

The instantaneous thrust coefficient is

$C_{\mathrm{T}_{\text {inst }}}=\frac{X^{\prime}}{\frac{1}{2} \rho V_{\infty}^{2}(2 r)}$ 


$$
=\left(\frac{W}{V_{\infty}}\right)^{2} \frac{c}{2 r}\left(c_{\mathrm{n}} \sin \theta-c_{\mathrm{t}} \frac{\cos \theta}{\cos \delta}\right),
$$

where the other normalization dimension comes from the distributed loads, which are a force per unit length in the $z$ direction. To get the total thrust coefficient we need to compute the azimuthal average:

$$
\begin{aligned}
C_{\mathrm{T}} & =\frac{B}{2 \pi} \int_{0}^{2 \pi} C_{\mathrm{T}_{\text {inst }}}(\theta) \mathrm{d} \theta \\
& =\frac{\sigma}{4 \pi} \int_{0}^{2 \pi}\left(\frac{W}{V_{\infty}}\right)^{2}\left(c_{\mathrm{n}} \sin \theta-c_{\mathrm{t}} \frac{\cos \theta}{\cos \delta}\right) \mathrm{d} \theta .
\end{aligned}
$$

From the thrust coefficient we can compute the expected induction factor by reversing Eq. (33):

$$
a= \begin{cases}\frac{1}{2}\left(1-\sqrt{1-C_{\mathrm{T}}}\right) & C_{\mathrm{T}} \leq 0.96 \text { (momentum) } \\ \frac{1}{7}\left(1+3 \sqrt{\frac{7}{2} C_{\mathrm{T}}-3}\right) & 0.96<C_{T}<2 \text { (empirical) } \\ \frac{1}{2}\left(1+\sqrt{1+C_{\mathrm{T}}}\right) & C_{\mathrm{T}}>2 \text { (propeller brake). }\end{cases}
$$

Finally, this induction factor allows us to compute the correction factor from Eq. (34). These factors should be multiplied against the induced velocities, but because that is the quantity we need to solve for, we must multiply against their predicted values.

Because this correction is derived for an isolated turbine, the correction factors $k_{1} \ldots k_{N}$ should be precomputed for each individual turbine in isolation rather than as part of the coupled solve of all turbines together.

\subsection{Matrix assembly and solution procedure}

From the proceeding discussion it should be noted that computing loads depends on the induced velocities, but computing the induced velocities depends on the loads. Thus, an iterative root-finding approach is required. We can assemble the self-induction and mutual induction effects into one large matrix composed of block matrices. We also need to apply the various correction factors $k$ for turbine $J$. To solve all induced velocities as one large system we will concatenate the $\boldsymbol{u}$ and $\boldsymbol{v}$ velocity vectors into one vector: $\boldsymbol{w}=[\boldsymbol{u} ; \boldsymbol{v}]$. In the equation below, the symbol $\odot$ represents an element-byelement multiplication.

$$
\left[\begin{array}{c}
\boldsymbol{u}_{1} \\
\boldsymbol{u}_{2} \\
\vdots \\
\boldsymbol{u}_{N} \\
-- \\
\boldsymbol{v}_{1} \\
\boldsymbol{v}_{2} \\
\vdots \\
\boldsymbol{v}_{N}
\end{array}\right]=\left[\begin{array}{c}
k_{1} \\
k_{2} \\
\vdots \\
k_{N} \\
-- \\
k_{1} \\
k_{2} \\
\vdots \\
k_{N}
\end{array}\right]
$$

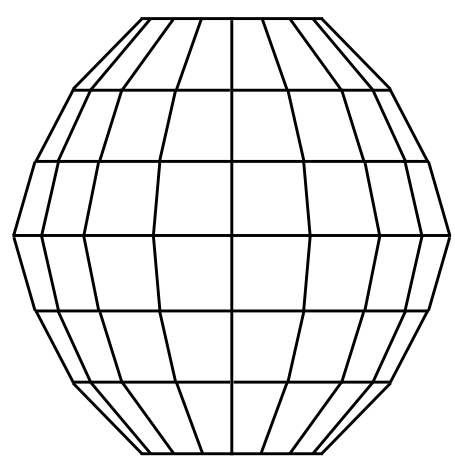

Figure 12. Example two-dimensional discretization in height and azimuthal position of swept surface (side view).

$\odot\left[\begin{array}{llll}\mathbf{A}_{x} & \mathbf{A}_{x_{12}} & \ldots & \mathbf{A}_{x_{1 N}} \\ \mathbf{A}_{x_{21}} & \mathbf{A}_{x} & \ldots & \mathbf{A}_{x_{2 N}} \\ \vdots & \vdots & \ddots & \vdots \\ \mathbf{A}_{x_{N 1}} & \mathbf{A}_{x_{N 2}} & \ldots & \mathbf{A}_{x} \\ \hdashline--- & ----- & -- & ---- \\ \mathbf{A}_{y} & \mathbf{A}_{y_{12}} & \ldots & \mathbf{A}_{y_{1 N}} \\ \mathbf{A}_{y_{21}} & \mathbf{A}_{y} & \ldots & \mathbf{A}_{y_{2 N}} \\ \vdots & \vdots & \vdots & \vdots \\ \mathbf{A}_{y_{N 1}} & \mathbf{A}_{y_{N 2}} & \ldots & \mathbf{A}_{y}\end{array}\right]\left[\begin{array}{c}\boldsymbol{q}_{1} \\ \boldsymbol{q}_{2} \\ \vdots \\ \boldsymbol{q}_{N}\end{array}\right]$

We now have a matrix vector expression of the form $\boldsymbol{w}=\mathbf{A} \boldsymbol{q}$, but because $\boldsymbol{q}$ depends on $\boldsymbol{w}$ we must solve for $\boldsymbol{w}$ using a root-finding method. The residual equation is

$f(\boldsymbol{w})=\mathbf{A} \boldsymbol{q}(\boldsymbol{w})-\boldsymbol{w}=0$.

Any good $n$-dimensional root finder can be used. This paper uses the modified Powell hybrid method as contained in hybrd.f of Minpack.

\subsection{Variations in height}

The actuator cylinder theory computes all loads in twodimensional cross sections. We can use a representative section to represent the whole turbine (which is more appropriate for an H-Darrieus geometry, ignoring wind shear), or we can additionally discretize the turbine along the height and compute loads at each section.

For each azimuthal station of interest, the solution is projected onto the instantaneous locations of the blade discretization as shown in Fig. 12. For an unswept blade, this involves just a straightforward transfer of forces as the blade discretization would typically be exactly aligned with the surface discretization. However, for swept blades, interpolation is necessary to resolve the forces along the curved blade path. Furthermore, for a swept blade, the normal and tangential directions change along the blade path. For swept blades, each point along the blade is at some azimuthal offset $(\Delta \theta)$ from a reference point (e.g., relative to the equatorial blade location), and the total normal force, tangential force, and torque produced by the blade are (again $\Delta \theta=0$ for unswept 
blades)

$$
\begin{aligned}
R_{\text {blade }}(\theta)= & \int\left[R^{\prime}(\theta+\Delta \theta) \cos (\Delta \theta)\right. \\
& \left.-T^{\prime}(\theta+\Delta \theta) \sin (\Delta \theta)\right] \mathrm{d} z \\
T_{\text {blade }}(\theta)= & \int\left[R^{\prime}(\theta+\Delta \theta) \sin (\Delta \theta)\right. \\
& \left.+T^{\prime}(\theta+\Delta \theta) \cos (\Delta \theta)\right] \mathrm{d} z \\
Z_{\text {blade }}(\theta)= & \int Z^{\prime}(\theta+\Delta \theta) \mathrm{d} z \\
Q_{\text {blade }}(\theta)= & \int r T^{\prime}(\theta+\Delta \theta) \mathrm{d} z .
\end{aligned}
$$

Now that the forces as a function of $\theta$ are known for one blade, the forces for all $B$ blades can be found. We let $\Delta \Theta_{j}$ represent the offset of blade $j$ relative to the first blade:

$\Delta \Theta_{j}=2 \pi(j-1) / B$.

The resulting forces in the inertial frame are then

$$
\begin{aligned}
X_{\text {all-blades }}(\theta)= & \sum_{j=1}^{B}-R_{\text {blade }}\left(\theta+\Delta \Theta_{j}\right) \sin \left(\theta+\Delta \Theta_{j}\right) \\
& -T_{\text {blade }}\left(\theta+\Delta \Theta_{j}\right) \cos \left(\theta+\Delta \Theta_{j}\right) \\
Y_{\text {all-blades }}(\theta)= & \sum_{j=1}^{B} R_{\text {blade }}\left(\theta+\Delta \Theta_{j}\right) \cos \left(\theta+\Delta \Theta_{j}\right) \\
& -T_{\text {blade }}\left(\theta+\Delta \Theta_{j}\right) \sin \left(\theta+\Delta \Theta_{j}\right) \\
Z_{\text {all-blades }}(\theta)= & \sum_{j=1}^{B} Z_{\text {blade }}\left(\theta+\Delta \Theta_{j}\right) .
\end{aligned}
$$

In this representation the velocities at each height can be different to account for wind shear or other wind distributions. This derivation is provided for completeness, but because of the increased computational expense, and to be consistent with the other comparisons we are making in this paper, we will focus on using one 2-D slice for the entire turbine.

\subsection{Power}

In addition to the thrust coefficient and instantaneous loads, which have already been defined, we are also interested in computing the power coefficient. This is easily computed from the instantaneous tangential load given in Eq. (27) (or Eq. 43). The torque (per unit length) is then

$Q=r T^{\prime}$

and the azimuthally averaged power is

$P=\frac{\Omega B}{2 \pi} \int_{0}^{2 \pi} Q(\theta) \mathrm{d} \theta$.
This is a periodic integral, and care should be taken in integrating near the boundaries because of the way the discretization is defined ( $\theta_{1}$ does not start at 0$)$. The power coefficient per unit length is then

$C_{\mathrm{P}}=\frac{P}{\frac{1}{2} \rho V_{\infty}^{3}(2 r)}$.

\subsection{Clockwise rotation}

The following derivation assumed counterclockwise rotation. For clockwise rotation a few minor changes must be made. Nothing in the influence coefficients needs changing as those are purely based on location. The only change for clockwise rotation is that the direction of $\hat{t}$ is reversed, as is the direction of the $\Omega r$ velocity vector in Figs. 8 and 9. The consequence is that the tangential velocity in Eq. (18) must be redefined as (note the two minus signs)

$V_{\mathrm{t}_{j}} \equiv-V_{\infty}\left(1+u_{j}\right) \cos \theta-V_{\infty} v_{j} \sin \theta+\Omega_{j} r_{j}$.

Additionally, the change in tangential direction affects the computation of the thrust coefficient. In Eq. (35) the sign is reversed on the second part of the equation. The consequence is that the total thrust coefficient (Eq. 37) would be computed as

$C_{\mathrm{T}}=\frac{\sigma}{4 \pi} \int_{0}^{2 \pi}\left(\frac{W}{V_{\infty}}\right)^{2}\left(c_{\mathrm{n}} \sin \theta+c_{\mathrm{t}} \frac{\cos \theta}{\cos \delta}\right) \mathrm{d} \theta$.

For transferring loads to an inertial frame, or for computing total blade loads with curved blades, a couple more changes are required. Equation (43) replaces the plus sign in front of $T_{\text {blade }}$ with a minus sign (for both the $X$ and $Y$ equation) and Eq. (41) is modified as

$$
\begin{aligned}
R_{\text {blade }}(\theta)= & \int\left[R^{\prime}(\theta+\Delta \theta) \cos (\Delta \theta)\right. \\
& \left.+T^{\prime}(\theta+\Delta \theta) \sin (\Delta \theta)\right] \mathrm{d} z \\
T_{\text {blade }}(\theta)= & \int\left[-R^{\prime}(\theta+\Delta \theta) \sin (\Delta \theta)\right. \\
& \left.+T^{\prime}(\theta+\Delta \theta) \cos (\Delta \theta)\right] \mathrm{d} z .
\end{aligned}
$$

\section{Two turbine interactions}

This methodology was implemented and made open-source (https://github.com/byuflowlab/vawt-ac) in Julia, which is a dynamic programming language designed for scientific computing (http://julialang.org). For single-turbine cases the implementation was verified against Madsen's results (Madsen et al., 2013). Our focus here is on multiple-turbine cases, and for simplicity on two turbine cases.

The first configuration we examine is the Mariah Windspire $1.2 \mathrm{~kW}$ VAWT, which has been the subject of multiple 
Table 1. Mariah Windspire $1.2 \mathrm{~kW}$ VAWT parameters.

\begin{tabular}{lr}
\hline Diameter & $0.6 \mathrm{~m}$ \\
Chord & $0.128 \mathrm{~m}$ \\
Number of blades & 3 \\
Height & $6.1 \mathrm{~m}$ \\
Airfoil & Du-06-W-200 \\
\hline
\end{tabular}

experimental and computational studies (Dabiri, 2011; Araya et al., 2014; Zanforlin and Nishino, 2016). Specifically, we compare against published 2-D unsteady Reynolds-averaged Navier-Stokes (URANS) simulations of two closely interacting turbines (Zanforlin and Nishino, 2016). This paper was one of the few interacting VAWT studies with sufficient detail to make a good comparison. The turbine parameters for this turbine are shown in Table 1. Wind tunnel data for the lift and drag coefficients of this airfoil are available for a few different conditions (Claessens, 2006).

Before comparing turbine pairs, we compared the performance of the isolated turbine to experimental data. The National Renewable Energy Laboratory (NREL) conducted field studies with the same turbine while installed at the National Wind Technology Center (Huskey et al., 2009). The isolated power coefficient and power, as a function of wind speed, are shown using the actuator cylinder method as compared to the experimental data in Fig. 13. The computational method overpredicts the power somewhat, which is not surprising considering that our 2-D simulations do not include the tower, struts, or tip losses and predict aerodynamic rather than electrical power. The published CFD data overpredict power by about the same percentage relative to experimental data, for similar reasons (Zanforlin and Nishino, 2016). We were not able to compare directly to the CFD data set, because the corresponding experimental data set from Windward Engineering was no longer available (in particular the rotation speed schedule was needed). Instead, we compared to the NREL data set, which tabulated the corresponding rotation speed for each wind speed. Overall, the agreement in the power performance trends is quite reasonable.

For a pair of closely spaced turbines we used the same conditions as in the CFD study, namely two identical turbines separated by two diameters (center to center), with the incoming wind direction varied around a full $360^{\circ}$. The tip-speed ratio was set at 2.625 for both turbines, which is an average of the two tip-speed ratios used in Zanforlin's study $(2.55,2.7$, which produce essentially the same results). The relative power of the pair of turbines, as compared to their power in isolation, is plotted versus the wind direction in Fig. 14. Also shown for comparison are the CFD results from the URANS study (Zanforlin and Nishino, 2016). While small differences exist, the overall agreement is very good. Benefits on the order of $5-10 \%$ are observed across a relatively wide range on inflow angles. These results are

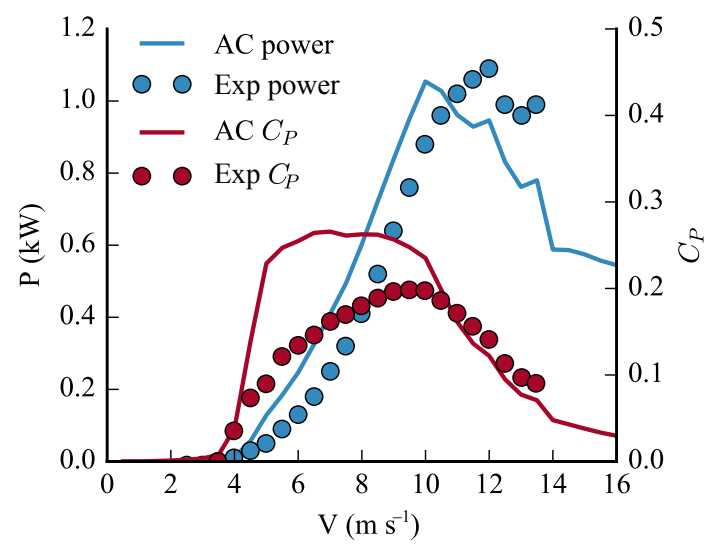

Figure 13. Power and power coefficient (separate $y$ axes), as a function of wind speed for the Windspire $1.2 \mathrm{~kW}$ turbine. Lines are from the actuator cylinder method (AC), and circles are from the NREL experimental data.

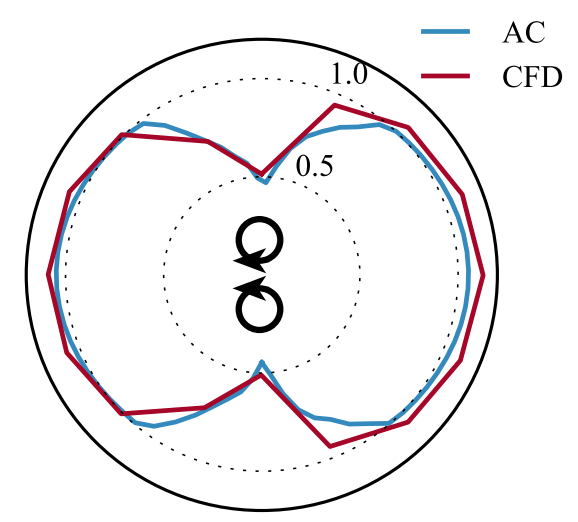

Figure 14. Normalized power as a function of wind direction on a polar plot, with an overlay of the turbine rotation directions. Results shown for both the actuator cylinder method (AC) and the CFD results of Zanforlin and Nishino (2016).

also in alignment with those observed experimentally for this same turbine (Dabiri, 2011).

Repeating this exercise for many different separations (e.g., two diameters was used in the previous case) requires a different visualization approach; otherwise, the plots overlap and become difficult to see. Instead of changing the wind direction for a fixed turbine position, we move the turbines around for a fixed wind position. The position of turbine 1 is fixed, and the position of turbine 2 is swept in concentric circles ranging from 1 diameter (no separation) to 6 diameters between turbine centers. The lower bound is of course unrealistically close, but because some VAWT concepts employ dual rotors with very close spacings, the smallest possible lower bound was used to observe trends across a broader range. By moving the turbines we can show the effect of relative separation and changing wind direction (via rotated positions) simultaneously. 


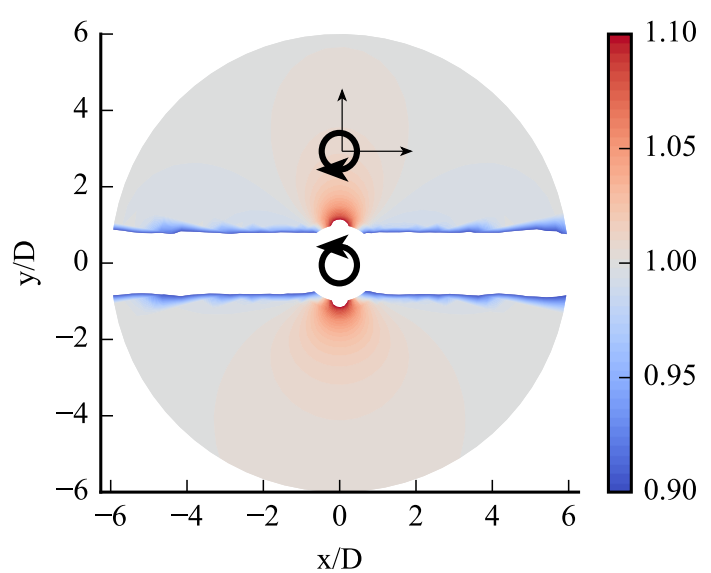

Figure 15. The first turbine is fixed in the center, and the second turbine is moved around in concentric circles with the wind incoming from the left. The contours show the normalized power of the two turbines (sum of their power normalized by sum of their power in isolation). The color scale is centered on 1.0 so that regions of beneficial interference can be more clearly seen. The color scale focuses on a small region $(0.9-1.1)$ for the same reason. The blank areas are waked regions, in which the power drops below the range of the color scale and so are not plotted.

Figure 15 shows the normalized power (total power of the turbines relative to their total power in isolation) as a function of the position of turbine 2 for counter-rotating turbines. Overlays of turbine 1 (fixed) and turbine 2 (position changed) are shown on the plots. The blank regions upstream and downstream of turbine 1 are regions where one of the turbines is in the other's wake. In these cases, the power drops by a large amount, well below the range of the color bars. The color bar range was chosen to highlight the mutual interference outside of the wake, which is of greater interest. We observe large regions of beneficial interference of around $5-10 \%$ for closely spaced turbines (with benefits exceeding $10 \%$ for very close spacings).

For counter rotating turbines, two configurations are possible: the Counter Up and the Counter Down configuration (Fig. 16). In Fig. 15 the upper half of the figure corresponds to the Counter Up configuration, and the lower half corresponds to the Counter Down configuration. For the current turbine and conditions, the Counter Down configuration shows somewhat larger benefits across a wider range of inflow angles, consistent with the reported CFD studies. A corotating configuration was also explored, which yielded very similar regions of beneficial interference. The figures were so similar in these conditions that a separate plot for the corotating case is not shown.

A second turbine and condition set came from a threedimensional incompressible Navier-Stokes simulation (Korobenko et al., 2013). This turbine was a $3.5 \mathrm{~kW}$ H-Darrieus from Cleanfield Energy Corporation. The turbine parameters are listed in Table 2. Wind tunnel data for the airfoil were ex-

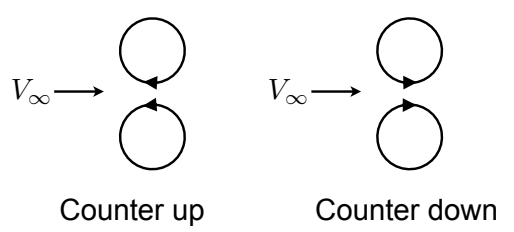

Figure 16. Two cases for a counter rotating pair of turbines. "Counter Up" refers to the pair with a rotation direction facing upstream at their closest interface, whereas the "Counter Down" configuration rotates downstream at their closest interface.

Table 2. Cleanfield $3.5 \mathrm{~kW}$ VAWT parameters.

\begin{tabular}{lr}
\hline Diameter & $2.5 \mathrm{~m}$ \\
Chord & $0.4 \mathrm{~m}$ \\
Number of blades & 3 \\
Height & $3 \mathrm{~m}$ \\
Airfoil & NACA 0015 \\
\hline
\end{tabular}

tracted from Sandia wind tunnel tests (Sheldahl and Klimas, 1981). The lift and drag coefficients change significantly at the Reynolds number of this turbine (275 000), and so to provide the best estimate possible the coefficient data were interpolated between the two nearest data sets: $R e=160000$ and $R e=360000$.

Because three-dimensional CFD is more computationally intensive, only one case was included in that study to compare against. That case consisted of two turbines in the Counter Down configuration, separated (tower to tower) by 2.64 rotor radii. The turbines were operated at a tip-speed ratio of 1.5 , which was the optimal condition for the singleturbine configuration. The CFD study reported a decrease in power for the counter-rotating pair, although the amount of decrease was not specified. Fortunately, torque vs. azimuth plots were presented. Extracting that data from the plot and integrating shows that the CFD simulations predicted a $12 \%$ decrease in power for the counter-rotating pair as compared to the isolated turbines.

Actuator cylinder theory was used for the same turbine at the optimal tip-speed ratio predicted using AC theory. A decrease in power for the Counter Down configuration was also observed of about $15 \%$. However, the optimal tip-speed ratio differed from the experimental data (Bravo et al., 2007). The nondimensional power curves were very similar, but with a shift in tip-speed ratio. To be consistent, the optimal tipspeed ratio was used in both cases ( 2.9 for the actuator cylinder case). Without more data to compare against, the robustness of this configuration to other conditions could not be assessed.

A third data set was examined, which came from wind tunnel tests of co- and counter-rotating VAWT pairs (AhmadiBaloutaki et al., 2015). This study used very high solidity rotors and found beneficial interference for counter-rotating turbines across all wind speeds tested. For co-rotating tur- 

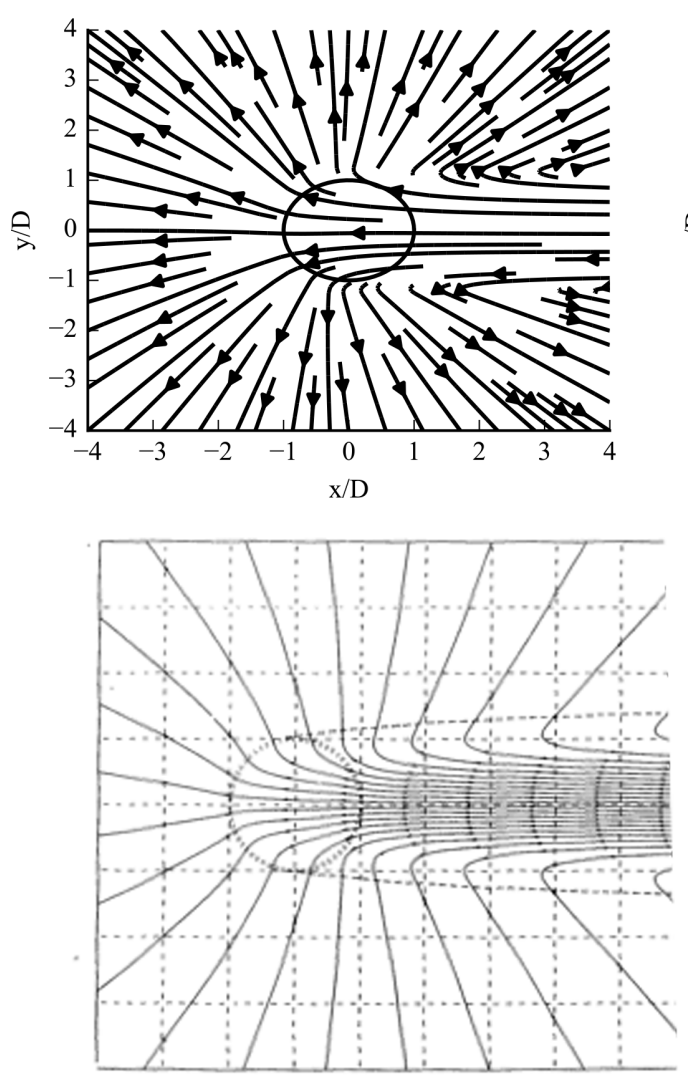
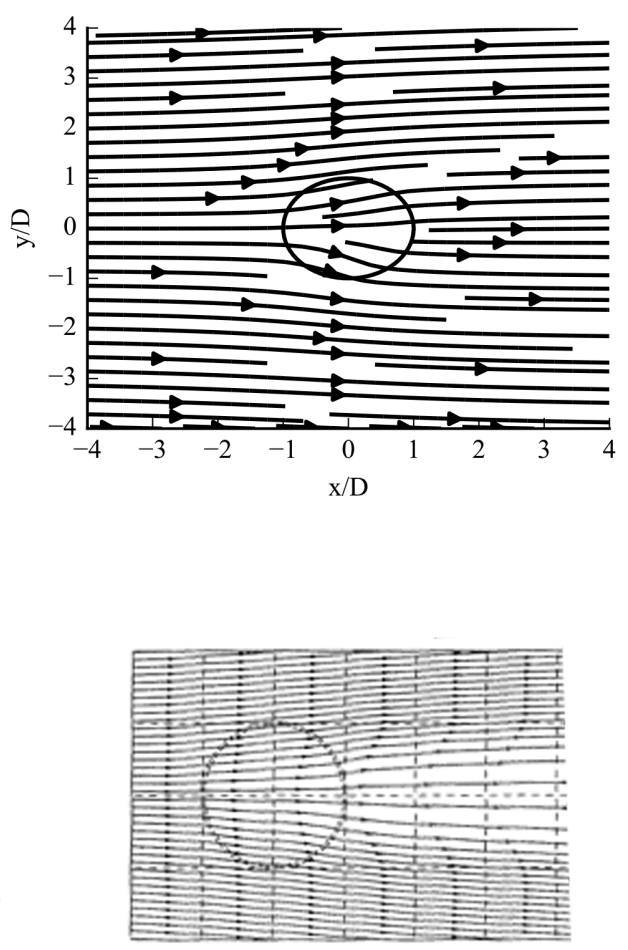

Figure 17. The top row is for the linear actuator cylinder theory, showing streamlines for an isolated turbine. The left panel shows the induced velocity only, while the right panel includes the freestream. The bottom row shows the same conditions, but with the full Euler equations (Madsen, 1982).

bines, beneficial interference was realized across some wind speeds, and detrimental interference at others. The turbines used in this wind tunnel study were inefficient under the conditions tested, with an extremely small power coefficient of $2 \times 10^{-3}$ (corresponding power of under $0.3 \mathrm{~W}$ ). The percent increase in power of turbine pairs was significant, but in absolute terms the power increase was very small. Similar relative benefits with actuator cylinder theory were observed depending on the assumed airfoil data. This turbine used a custom-made airfoil section and, without corresponding wind tunnel data, detailed comparisons to actuator cylinder theory were not possible.

In addition to comparing power output, we compared the induced velocity field generated by the VAWT. Ultimately, this induced velocity is what changes the incoming flow field for a second turbine. The streamlines for the induced velocity field of the isolated VAWT and the total velocity field (with freestream added) are shown in Fig. 17. For comparison, the streamlines for a solution based on the full Euler equations are shown below (Madsen, 1982). Although the actuator cylinder theory uses a linearization and only adds a nonlinear correction, the induced and total velocity fields compare well to those from the Euler equations. These in- duced velocities were also found to compare well to that from an unsteady panel simulation of a VAWT (see Figs. 3.7 and 3.8 in Ferreira, 2009).

A properly positioned pair of VAWTs can produce power increases, but one must be careful in extrapolating results to the wind farm. Even in just the two-turbine case, the overall benefits may or may not be realized depending on the site conditions. Although power increases of around 5-10\% may be realized across a wide range of inflow angles, for inflow angles that create wake interference the power loss can drop by $50 \%$. As an example, assuming a uniform wind rose (wind equally probable in all directions), the data in Fig. 14 yield an expected value of power of around $90 \%$ of that in isolation (both using this method and the CFD results). In other words, with a uniform wind rose the wake losses overwhelm the benefits of close spacing. A more directional wind rose, on the other hand, could realize overall power increases.

Additionally, a wind farm consists of many turbines, and accounting for wake effects across the full wind rose is essential. VAWTs tend to produce shorter wakes than HAWTs, which can help alleviate some of the spacing challenges. However, if turbines are intentionally positioned close together to create beneficial interference, then they also have 
the potential to create strong power losses through wake effects.

Beneficial interference may be possible not just for pairs of turbines, but for carefully positioned arrays of turbines, as demonstrated in 2-D URANS simulations (Bremseth and Duraisamy, 2016). However, the benefits have only been explored with one wind direction. Our past research in HAWT wind farm optimization suggests that when optimizing turbine positioning under uncertainty of wind direction, the optimal configurations spread out and are not in aligned rows in order to minimize wake interference (Fleming et al., 2016; Gebraad et al., 2016). Further investigation is needed to better understand how to optimize VAWT farms.

\section{Conclusions}

Actuator cylinder theory is a fast and often effective analysis method to predict aerodynamic loads and power of vertical axis wind turbines. In this paper we derive an extension to actuator cylinder theory for multiple interacting turbines. Additional extensions were provided that apply to both the singleturbine and multiple-turbine cases: thrust coefficients outside of the momentum regions, and curved or swept blades.

Comparisons to published data for VAWT pairs showed reasonable agreement in predicting relative power changes and induced velocities using actuator cylinder theory. However, more data are needed to better assess the conditions under which the multiple actuator cylinder theory is effective. Like blade element momentum methods, the accuracy and usefulness of actuator cylinder theory is highly dependent on providing accurate airfoil coefficient data.

For closely spaced VAWTs, we observed cases with beneficial interference across a wide range of inflow angles, with peak power increases exceeding $10 \%$. In other cases, detrimental interference was observed. The conditions that affect the interactions depend on many things, including the airfoil aerodynamic performance, the tip-speed ratios, and solidities. Our focus here is to demonstrate the actuator cylinder theory and not to make a thorough investigation of closely interacting VAWTs, but more illumination on this topic is needed.

Further improvements to actuator cylinder theory are needed, as are more detailed validation studies. An improved wake model is necessary because the wakes in actuator cylinder theory are inviscid, do not decay, and do not spread. Our recent work has developed the first VAWT wake model derived from computational fluid dynamics simulations that is parametrized for turbines with different tip-speed ratios and solidities (Tingey and Ning, 2016). Models like this could be combined with actuator cylinder theory to better predict turbine-wake interactions.

Because of the model's sensitivity to the airfoil data, further data collection and improved methods of applying rotational corrections specific to VAWTs may be needed. Al- though the method was only demonstrated in 2-D, as outlined in the methods section, extensions to 3-D are straightforward using a strip-wise approach, but tip-loss corrections should be added. Additionally, appropriate methods for accounting for losses through struts and the tower will likely improve aerodynamic performance estimates.

\section{Data availability}

Source code for my implementation of this actuator cylinder method has been made publicly available (Ning, 2016a), as well as code and supplementary files used to generate the data figures in this paper (Ning, 2016b).

Edited by: J. Meyers

Reviewed by: two anonymous referees

\section{References}

Ahmadi-Baloutaki, M., Carriveau, R., and Ting, D. S.-K.: Performance of a vertical axis wind turbine in grid generated turbulence, Sustainable Energy Technologies and Assessments, 11, 178-185, doi:10.1016/j.seta.2014.12.007, 2015.

Araya, D. B., Craig, A. E., Kinzel, M., and Dabiri, J. O.: Low-order modeling of wind farm aerodynamics using leaky Rankine bodies, Journal of Renewable and Sustainable Energy, 6, 063118, doi:10.1063/1.4905127, 2014.

Bravo, R., Tullis, S., and Ziada, S.: Performance testing of a small vertical-axis wind turbine, in: Proceedings of the 21st Canadian Congress of Applied Mechanics (CANCAM07), 3-7 June 2007, Toronto, Canada, 2007.

Bremseth, J. and Duraisamy, K.: Computational analysis of vertical axis wind turbine arrays, Theor. Comp. Fluid Dyn., 30, 387-401, doi:10.1007/s00162-016-0384-y, 2016.

Buhl Jr., M. L.: A New Empirical Relationship between Thrust Coefficient and Induction Factor for the Turbulent Windmill State, National Renewable Energy Laboratory, Technical Report, NREL/TP-500-36834, 7 pp., 2005.

Burton, T., Jenkins, N., Sharpe, D., and Bossanyi, E.: Wind Energy Handbook, 2nd Edn., Wiley, UK, 2011.

Claessens, M.: The Design and Testing of Airfoils for Application in Small Vertical Axis Wind Turbines, Master's thesis, Delft University of Technology, the Netherlands, 2006.

Dabiri, J. O.: Potential Order-of-Magnitude Enhancement of Wind Farm Power Density Via Counter-Rotating Vertical-Axis Wind Turbine Arrays, Journal of Renewable and Sustainable Energy, 3, 043104, doi:10.1063/1.3608170, 2011.

Ferreira, C. S.: The Near Wake of the Vawt, PhD thesis, Delft University of Technology, the Netherlands, 2009.

Ferreira, C. S., Madsen, H. A., Barone, M., Roscher, B., Deglaire, P., and Arduin, I.: Comparison of Aerodynamic Models for Vertical Axis Wind Turbines, J. Phys. Conf. Ser., 524, 012125, doi:10.1088/1742-6596/524/1/012125, 2014.

Fleming, P., Ning, A., Gebraad, P., and Dykes, K.: Wind Plant System Engineering through Optimization of Layout and Yaw Control, Wind Energy, 19, 329-344, doi:10.1002/we.1836, 2016.

Gebraad, P., Thomas, J. J., Ning, A., Fleming, P., and Dykes, K.: Maximization of the Annual Energy Production of Wind Power 
Plants by Optimization of Layout and Yaw-Based Wake Control, Wind Energy, doi:10.1002/we.1993, online first, 2016.

Hansen, M. O. L.: Aerodynamics of Wind Turbines, 2nd Edn., Earthscan, London, UK, 2008.

Huskey, A., Bowen, A., and Jager, D.: Wind Turbine Generator System Power Performance Test Report for the Mariah Windspire 1-kW Wind Turbine, National Renewable Energy Laboratory, Technical Report, NREL/TP-500-46192, doi:10.2172/969714, 2009.

Korobenko, A., Hsu, M.-C., Akkerman, I., and Bazilevs, Y.: Aerodynamic Simulation of Vertical-Axis Wind Turbines, J. Appl. Mech., 81, 021011, doi:10.1115/1.4024415, 2013.

Madsen, H.: The Actuator Cylinder - a Flow Model for Vertical Axis Wind Turbines, PhD thesis, Aalborg University Centre, 1982.

Madsen, H., Larsen, T., Vita, L., and Paulsen, U.: Implementation of the Actuator Cylinder Flow Model in the HAWC2 Code for Aeroelastic Simulations on Vertical Axis Wind Turbines, in: 51st AIAA Aerospace Sciences Meeting, 7-10 January 2013, Grapevine, Texas, AIAA 2013-0913, doi:10.2514/6.2013-913, 2013.

Manwell, J. F., Mcgowan, J. G., and Rogers, A. L.: Wind Energy Explained, 2nd Edn., Wiley, UK, 2009.

Ning, A.: A Simple Solution Method for the Blade Element Momentum Equations with Guaranteed Convergence, Wind Energy, 17, 1327-1345, doi:10.1002/we.1636, 2014.

Ning, A.: VAWT AC, Zenodo, doi:10.5281/zenodo.165183, 2016a.

Ning, A.: VAWT AC Supplementary Files, figshare, doi:10.6084/m9.figshare.3382504.v3, 2016b.

Paraschivoiu, I.: Double Multiple Streamtube Model for Darrieus Wind Turbines, in: 2nd DOE/NASA Wind Turbine Dynamics Workshop, 24-26 February 1981, Cleveland, OH, 1981.
Paraschivoiu, I.: Double-multiple streamtube model for studying vertical-axis wind turbines, J. Propul. Power, 4, 370-377, doi:10.2514/3.23076, 1988.

Paraschivoiu, I. and Delclaux, F.: Double Multiple Streamtube Model with Recent Improvements (for Predicting Aerodynamic Loads and Performance of Darrieus Vertical Axis Wind Turbines), J. Energy, 7, 250-255, doi:10.2514/3.48077, 1983.

Sheldahl, R. E. and Klimas, P. C.: Aerodynamic characteristics of seven symmetrical airfoil sections through 180-degree angle of attack for use in aerodynamic analysis of vertical axis wind turbines, Sandia National Labs., Albuquerque, NM, USA, Technical Report, SAND80-2114, doi:10.2172/6548367, 1981.

Strickland, J.: A performance prediction model for the Darrieus turbine, in: International Symposium on Wind Energy Systems, 1, 3 pp., 1975.

Templin, R.: Aerodynamic performance theory for the NRC vertical-axis wind turbine, LTR-LA-160, National Aeronautical Establishment, Ottawa, Ontario, Canada, 1974.

Tingey, E. and Ning, A.: Parameterized Vertical-Axis Wind Turbine Wake Model Using CFD Vorticity Data, in: ASME Wind Energy Symposium, 4-8 January 2016, San Diego, CA, doi:10.2514/6.2016-1730, 2016.

Wilson, R. and Lissaman, P.: Applied Aerodynamics of Wind Power Machines, Oregon State University, Tech. rep., 109 pp., 1974.

Zanforlin, S. and Nishino, T.: Fluid dynamic mechanisms of enhanced power generation by closely spaced vertical axis wind turbines, Renew. Energ., 99, 1213-1226, doi:10.1016/j.renene.2016.08.015, 2016. 